\title{
Effects of immediate repetition at different stages of consecutive interpreting training
}

\author{
An experimental study
}

\author{
Wenting $\mathrm{Yu}^{1,2}$ and Vincent J. van Heuven ${ }^{2}$ \\ ${ }^{1}$ Shanghai International Studies University / ${ }^{2}$ Leiden University
}

\begin{abstract}
The present study investigates whether immediate repetition improves consecutive interpreting performance during training. In addition, the study tries to shed light on whether the effects of immediate repetition differ between BA and MA interpreting trainees. In the experiment, ten raters judged six major quality measures of the accuracy and fluency of the interpreting output recorded from seven BA trainees and five MA trainees. The seventh quality measure expressed linguistic complexity as the number of clauses per AS-unit. The results show that the main effects of repetition and proficiency are both significant on accuracy and fluency, but the main effects are absent on linguistic complexity. Moreover, in terms of fluency BA trainees benefit significantly more from repetition than MA trainees. Accuracy improvement through repetition does not differ significantly between the two groups. The results have implications for consecutive interpreting training at different stages.
\end{abstract}

Keywords: effects of immediate repetition; consecutive interpreting training; training stages

\section{Introduction}

Previous research shows that repetition improves the oral performance of L2 learners (see for instance Bygate 1996, 2001; Lynch \& Maclean 2000, 2001). This observation supports early behaviorist psychology, as represented, for instance, by Skinner (1953) and Pavlov (1957), which dominated early language learning theories. It was assumed that language is learned via a process of habit formation through repetition (see for instance Paulston \& Bruder 1976). Bygate (2006) enriched the concept of repetition in language learning by emphasizing the repetition of familiar content and form. This new conceptualization is partly informed 
by the view that our attentional and processing capacity during communication activities is inherently restricted. For instance, L2 learners cannot focus on meaning and form simultaneously (Ahmadian 2012). Performing the same task again, therefore, might enable L2 learners to free mental resources gained from planning the content of communication, the access and formulation of words and grammatical structures during the first delivery. However, no parallel study has ever examined the effects of repetition on the cognitively more complicated language task of interpreting, which requires not only L2 oral proficiency but also interpreting skills.

In this paper, we investigate the effects of repetition in consecutive interpreting (CI) training (in this study L1 Chinese interpreted into L2 English). Specifically, we will explore (1) whether immediate repetition may improve the quality of CI during training, and if so, in terms of what aspect(s); and (2) whether the effects of repetition might vary with proficiency. Examining such effects would help CI instructors to assess the potential of repetition as an effective pedagogical approach to improve specific quality aspects during different stages of CI training.

\subsection{Repetition as a task-based pedagogical approach to improve L2 oral performance}

There has been abundant research on pedagogy of L2 oral proficiency training, focusing on the effects of learning context (immersion, studying abroad, and language instruction in the home country), planning (strategic planning and on-line planning), task repetition, and language instruction (see for instance Foster \& Skehan 1996; Towell 2002; Freed, Segalowitz \& Dewey 2004; Segalowitz \& Freed 2004). Task repetition is defined as "repetitions of the same or slightly altered tasks - whether whole tasks, or parts of a task" (Bygate \& Samuda 2005). Bygate's (1996, 2001) studies on task repetition have revived the research into the area of task repetition and its pedagogical value in L2 oral proficiency (see for instance Lynch \& Maclean 2000, 2001; Ahmadian \& Tavakoli 2010; de Jong \& Perfetti 2011). These studies analyzed the impact of repetition on some (or all) of the three oral L2 quality aspects: Fluency, accuracy and complexity. Bygate (1996) found that repeating an L2 retelling task led to improved fluency, accuracy, and complexity. Bygate (2001) reported higher complexity in the repeated delivery of an L2 narrative task but also noticed a trade-off with accuracy and fluency. Lynch and Maclean (2000, 2001), in a study on the effect of repetition in an L2 speech task of poster presentation, found that immediate repetition improved accuracy in terms of phonology, vocabulary, semantic precision, and syntax. Meanwhile, they presented evidence for gains in fluency. De Jong and Perfetti (2011) had two groups of subjects repeat an L2 monologue task, with one group speaking on the same topic three times, 
and the other group on three different topics. Fluency improved for both groups during training but was maintained on post-tests only by the students who had repeatedly spoken on the same topic.

To summarize, task repetition facilitates L2 oral performance in a general sense in that the speaker can free processing capacity by building on the work of conceptualization, formulation, and articulation during the first delivery and shift attention to different aspects of the oral production process on a later attempt. Drawing on Levelt's (1989) speech production model, which comprises three stages, i.e. conceptualisation, formulation, and articulation, task repetition has the potential to (i) improve and speed up the recall and organization of conceptual ideas through conceptual priming, (ii) lead to speedier and more accurate and sophisticated formulation via strengthened links between conceptual content and lexicogrammatical forms through semantic, lexical and syntactic priming and increased capacity for lexico-grammatical searches to refine previous formulations, and (iii) implement faster and more accurate realization of the phonetic plan through phonological priming. However, there is lack of agreement in different studies across different types of L2 oral tasks on whether improvement was observed in accuracy, fluency or complexity concurrently with repeated attempts. Moreover, different ways were adopted to measure accuracy, fluency and complexity, all based on textual analyses. Hardly any study of this kind (oral communication in nature) used subjective rating methods, or a combination of textual analyses with subjective rating to assess quality of L2 oral performance. Finally, there are hardly any studies on whether the effect of repetition varies with L2 proficiency. These aspects will be taken into account in this study.

\subsection{Consecutive interpreting training}

\subsubsection{Consecutive interpreting}

$\mathrm{CI}$ is a form of interpreting in which the speaker and interpreter alternate when speaking, in contrast to simultaneous interpreting (SI), in which the speaker and interpreter speak at the same time. According to Gile (1995), interpreter training should start with CI, which naturally promotes sound interpreting methods, and should be considered the foundation for later SI training.

The perception and expectation of interpretation quality is generally agreed to involve content (accuracy and completeness of information conveyance, correct grammar, complexity and refinement of diction, logical cohesion, correct terminology) and form (fluency of delivery, accents, intonation, voice quality) (see for instance Bühler 1986; Zwischenberger \& Pöchhacker 2010).

The Association Internationale des Interprètes de Conférence (AIIC), the organization to which individual practitioners and schools of interpretation refer 
for guidance, states criteria regarding interpreters' working languages, which are classified into active (A language and B language) and passive (C language). An 'A' language is the interpreter's native or other language strictly equivalent to a native language into which interpreters can work. A ' $\mathrm{B}$ ' language is the language other than the interpreter's native language of which he has a perfect command and into which he can work. 'C' languages are those of which the interpreter has a complete understanding and from which he works (AIIC 2002).

Most Chinese native interpreters, who have Chinese as A language and English as $B$ language, are required to interpret both into and from either of the two active languages. For most beginning interpreting trainees who are not early bilinguals, the imbalance of their two working languages always poses a major challenge. Therefore, linguistic refinement, in particular that of L2 oral proficiency, is often given much attention throughout the entire interpreting training program.

\subsubsection{Cognitive effort model of CI processing for training purposes}

Gile (1995) proposed the Cognitive Effort Model of CI, with two performing phases: (1) the listening and note-taking phase, and (2) the speech production phase. This model is based on the assumption that the operations of interpretation are nonautomatic and compete for limited attentional resources. Therefore there is a need to explore the relationship between processing capacity requirements and limitations.

Phase One: $\mathrm{CI}=\mathrm{L}+\mathrm{M}+\mathrm{N}+\mathrm{C}$

Phase Two: $\mathrm{CI}=\mathrm{Rem}+\operatorname{Read}+\mathrm{P}$

In Phase One, the Listening and Analysis Effort (L) attends to the perception and analysis of the source language, which entail the identification of syllables and words, and the semantic decisions. The Short Term Memory Effort (M) stores and processes information in and from memory until it is organized into structures of expression. The Note-taking Effort $(\mathrm{N})$, an information analysis process itself, involves the selection and noting down of key information to trigger later recall. The Coordination Effort (C) coordinates the other three overlapping efforts in Phase One to prevent them from reaching saturation.

In Phase Two, the Remembering Effort (Rem) involves the long-term memory functions for information retrieval. Note-reading (Read) serves to trigger shortterm memory. The Production Effort (P) is associated with the formulation and articulation of structured natural language based on the retrieved messages and self-monitoring.

Gile (1995) emphasized that for successful CI performance, the processing capacity requirement for each effort should not exceed the capacity available for 
that effort and the total requirements of processing capacity for all efforts in each phase should not exceed the total available capacity.

Summarizing, CI is a cognitively more demanding language task than L2 oral tasks, involving both oral L2 communication and the interpreting skills. The biggest difference between CI and L2 speech production lies in the source for formulation and articulation stages. In contrast to the conceptualization stage in L2 speech production, CI starts with perception and comprehension of the source language, paralleled with storage, processing and retrieval of information through note-taking, memory functions and coordination of all these efforts. As a result, attentional resources are subject to fiercer competition in CI than L2 speech production. Interpreters may benefit more from freeing up processing capacity.

\subsection{Hypothesis and research questions}

This paper posits a general hypothesis that immediate repetition improves the performance of the cognitively more demanding language task of CI. Two research questions, as shown below, are to be investigated for the verification of the hypothesis:

1. Does immediate repetition improve the main quality aspects of CI during training (L1 interpreted into L2), and if so, in what aspect(s)?

2. Do the effects of repetition, if there are any, differ between relatively highly proficient (MA level) and relatively low proficient (BA level) interpreting trainees?

\section{Methods}

\subsection{Participants}

Twelve students from Shanghai International Studies University studying in two interpreting training programs (BA level and MA level) participated in this study. There were seven third-year BA level translation and interpretation majors with a mean age of 20. There were five second-year MA students with a mean age of 25 from the Graduate Institute of Interpretation and Translation. The BA students were oriented towards the development of basic interpreting skills while the MA students were oriented towards becoming professional conference interpreters. By the time of the experiment, the BA students had completed three basic CI training courses in three semesters while the MA students, all of whom were already part-time conference interpreters, had completed three semesters of intensive and 
advanced CI and SI training (minimally three hours training a day). All participants speak Chinese as their L1 and English as their L2.

\subsection{Materials}

A source audio clip in Chinese (lasting 3.5 minutes with a total of 501 Chinese characters comprising six parts) was edited on the basis of the press conference held by the former Chinese Premier Wen Jiabao during the National People's Congress in 2009. The audio clip is intended for the student participants to interpret into the target language of English. Of the six interpreted parts, two (Parts 4 and 5) were selected for rating and analysis because the whole rating task had to be performed within manageable time to avoid rater fatigue.

\subsection{Procedures}

The experiments for the two groups of participants took place on separate days in conference rooms equipped with booths for simultaneous interpreting. The source stimulus material and the participants' rendition were recorded synchronously on separate tracks. The experimenter monitored the participants' performance over headphones and ensured that all participants would finish interpreting the previous part before a new one was played to them. Task instructions were introduced before the experiments started. All participants were asked to interpret the same source speech three times (Delivery 1, $2 \& 3$ ) part by part with a short break of two minutes between deliveries. Deliveries 1 and 3 were selected for both auditory rating and text analysis. Delivery 2 was excluded because previous studies suggest that the third delivery is often the optimal during oral task repetition (see for instance Zhou 2006).

The online survey software of Qualtrics was used for the rating procedure. Twenty-four uploaded clips (12 interpreters $\times 2$ deliveries) were rated on six measures related to accuracy and fluency as the main quality aspects of interpretation, i.e. (i) accuracy of information, (ii) accuracy of grammar, (iii) speed of delivery, (iv) control of pauses, (v) control of other disfluencies (unnecessary repetition, false starts, inappropriate syllable lengthening, and self-corrections), and (vi) overall fluency, on a scale between 1 and 10. The presentation of the 24 clips was randomized for each rater. Ten raters, who studied, are studying or teaching at Leiden University, participated in the online rating. Three raters were native English speakers (two British English speakers and one American English speaker) and seven were highly proficient English speakers (six L1 Dutch speakers and one L1 Portuguese speaker); five were females and five were males. The raters' average number of experiences of attending interpreter-mediated events was 2 . 
The raters were informed that the whole rating session would last for an hour and they were recommended to take a ten-minute break after having rated 12 clips to avoid fatigue. They were then asked to complete a background survey, and to carefully read through the reference translation version of the two parts in the target language of English to understand the messages that the students were asked to interpret. Subsequently, two sample audio clips recorded prior to the experiment were played to them, one representing a highly proficient interpretation of the stimulus passage and the other a less proficient rendition.

All 24 clips were transcribed into text by a graduate student assistant and were checked by the author. The transcription was analyzed to assess linguistic complexity, another quality aspect of interpretation. Linguistic complexity is expressed as the ratio of the number of clauses relative to per AS-unit (an index of the degree of clause embedding) and is calculated by dividing the number of clauses by the number of AS-units. An AS-unit (Analysis of Speech Unit), is defined as a single speaker's utterance consisting of either an independent clause (minimally a clause including a finite verb), or sub-clausal unit, together with any subordinate clause(s) associated with it (Foster, Tonkyn \& Wigglesworth 2000). It is mainly a syntactic unit for analyzing spoken language. The more (embedded) clauses one AS-unit contains, the larger the ratio gets, and complexity increases. Clauses that were disfluent in nature, such as repetitions or false starts, were excluded; only the final corrected clauses were taken into account.

\subsection{Data analysis}

The ten raters were highly consistent in their ratings on the six measures related to accuracy and fluency, with a mean Cronbach's alpha of .96 (the minimal alpha is .94 for accuracy of information). The complexity assessment was conducted by the author and checked by a student assistant, with a high agreement rate of $96 \%$.

For the statistical analyses of the rating results and textual analysis, repeatedmeasures Analysis of Variance (RM-ANOVA) and paired $t$-tests were performed. Delivery (Delivery $1 \& 3$ ) was a within-subjects factor and interpreting proficiency (BA level \& MA level) a between-subjects variable. 


\section{Results}

3.1 Six measures related to accuracy and fluency

The mean ratings on the six measures related to accuracy and fluency are presented in Table 1. The main effects of proficiency and repetition and interaction are summarized in Table 2.

Table 1. Means of the ratings of the six measures related to accuracy and fluency (PR = Proficiency, ID = Identification number of participants, AI = Accuracy of Information, $\mathrm{AG}=$ Accuracy of Grammar, $\mathrm{SD}=$ Speed of delivery, $\mathrm{CP}=$ Control of pauses, $\mathrm{CD}=$ Control of other disfluencies, $\mathrm{OF}=$ Overall fluency).

\begin{tabular}{lccccccccccccc}
\hline \multicolumn{1}{c}{} & \multicolumn{1}{c}{ Delivery 1 } & \multicolumn{1}{c}{ Delivery 3} \\
\hline PR & ID & AI & AG & SD & CP & CD & OF & AI & AG & SD & CP & CD & OF \\
BA & 1 & 5.7 & 5.7 & 5.7 & 4.8 & 4.8 & 5.3 & 6.1 & 6.4 & 6.7 & 6.1 & 5.4 & 6.1 \\
BA & 2 & 5.6 & 5.2 & 5.5 & 4.9 & 5.0 & 5.6 & 7.5 & 6.6 & 6.5 & 5.5 & 5.6 & 6.3 \\
BA & 3 & 4.5 & 4.6 & 5.0 & 4.7 & 4.3 & 4.5 & 6.4 & 6.0 & 6.2 & 5.9 & 5.9 & 6.2 \\
BA & 4 & 5.2 & 4.9 & 4.4 & 4.0 & 3.6 & 4.1 & 5.1 & 5.2 & 5.9 & 5.6 & 5.2 & 5.7 \\
BA & 5 & 5.6 & 5.3 & 5.2 & 5.0 & 4.8 & 5.1 & 6.8 & 6.6 & 6.1 & 6.4 & 6.0 & 6.2 \\
BA & 6 & 4.7 & 5.2 & 4.9 & 4.6 & 4.4 & 4.8 & 6.7 & 5.9 & 5.5 & 5.6 & 6.0 & 5.9 \\
BA & 7 & 5.5 & 5.7 & 5.0 & 3.9 & 4.4 & 4.4 & 5.7 & 6.0 & 6.3 & 5.7 & 5.1 & 5.7 \\
Mean BA & 5.3 & 5.2 & 5.1 & 4.6 & 4.5 & 4.8 & 6.3 & 6.1 & 6.2 & 5.8 & 5.6 & 6.0 \\
MA & 8 & 7.0 & 6.6 & 7.0 & 6.4 & 6.1 & 6.8 & 7.5 & 6.5 & 7.3 & 6.9 & 6.5 & 7.2 \\
MA & 9 & 7.6 & 6.8 & 7.7 & 6.7 & 6.4 & 7.1 & 8.1 & 7.6 & 7.9 & 7.6 & 7.4 & 7.6 \\
MA & 10 & 7.8 & 6.9 & 7.6 & 6.9 & 6.8 & 7.3 & 8.2 & 7.7 & 8.0 & 7.2 & 7.2 & 7.8 \\
MA & 11 & 7.1 & 5.7 & 6.3 & 5.7 & 5.7 & 6.1 & 7.4 & 6.0 & 6.8 & 6.6 & 6.2 & 6.6 \\
MA & 12 & 5.6 & 5.5 & 5.6 & 4.8 & 4.7 & 5.3 & 6.2 & 6.7 & 6.7 & 5.8 & 5.2 & 5.8 \\
Mean MA & 7.0 & 6.3 & 6.8 & 6.1 & 5.9 & 6.5 & 7.5 & 6.9 & 7.3 & 6.8 & 6.5 & 7.0 \\
Grand mean & 6.0 & 5.7 & 5.8 & 5.2 & 5.1 & 5.5 & 6.8 & 6.4 & 6.7 & 6.2 & 6.0 & 6.4 \\
\hline
\end{tabular}

Table 2. Summary of RM-ANOVA. F-ratio, p-value and effect size (partial eta squared) for main effects of proficiency and repetition and of interaction. For all effects and interactions: $\mathrm{df} 1=1, \mathrm{df} 2=10$.

\begin{tabular}{lllllllllll}
\hline Judgment measures (down) & \multicolumn{3}{l}{ Proficiency } & \multicolumn{4}{c}{ Repetition } & \multicolumn{3}{c}{ Interaction $\mathrm{p} \times \mathbf{r}$} \\
\cline { 2 - 11 } & $\mathrm{F}$ & $\mathrm{p}$ & $\mathrm{p} \eta^{2}$ & $\mathrm{~F}$ & $\mathrm{p}$ & $\mathrm{p} \eta^{2}$ & $\mathrm{~F}$ & $\mathrm{p}$ & $\mathrm{p} \eta^{2}$ \\
\hline Accuracy of information & 15.2 & .003 & .603 & 14.0 & .004 & .583 & 2.2 & .166 & .182 \\
Accuracy of grammar & 10.2 & .010 & .504 & 25.5 & $<.001$ & .718 & $<1$ & & \\
Speed of delivery & 20.1 & .001 & .668 & 71.0 & $<.001$ & .877 & 9.4 & .012 & .484 \\
Control of pauses & 15.9 & .003 & .613 & 88.8 & $<.001$ & .899 & 6.8 & .026 & .405 \\
Other disfluencies & 11.7 & .006 & .540 & 49.9 & $<.001$ & .833 & 5.7 & .039 & .361 \\
Overall fluency & 15.0 & .003 & .599 & 94.5 & $<.001$ & .904 & 17.0 & .002 & .629 \\
\hline
\end{tabular}


The interactions between proficiency and repetition are visualized in Figure 1. Both factors in the design, i.e. proficiency (BA level, MA level) and repetition (Delivery 1,3 ), are significant for each of the six judgment measures of accuracy and fluency. As shown in Figure 1, the effect is - predictably - that the MA trainees outperform the BA trainees, and that the performance is judged more favorably on the third attempt than on the first. The effect of repetition is generally stronger than that of proficiency.

We may observe, next, that the effect of repetition is larger for BA trainees than for MA trainees (for details see Figure 1). This interaction effect, however, is insignificant in the case of accuracy of grammar and accuracy of information.

The results of paired $t$-tests comparing Delivery 1 and 3 within each group are presented in Table 3. BA trainees made significant improvement on all six measures in Delivery 3 over Delivery 1, while MA trainees made significant improvement on all measures except accuracy of grammar.

Table 3. $t$-tests (comparing Delivery 1 with Delivery 3 within BA group and MA group respectively). The Table specifies $t$ and p-values (two-tailed).

\begin{tabular}{lllll}
\hline Judgment measures (down) & \multicolumn{2}{c}{ BA (Delivery 1 vs. 3) } & \multicolumn{2}{c}{ MA (Delivery 1 vs. 3) } \\
\cline { 2 - 5 } & $\mathrm{t}(6)$ & $\mathrm{p}$ & $\mathrm{t}(4)$ & $\mathrm{p}$ \\
\hline Accuracy of information & -3.2 & .020 & -9.0 & .001 \\
Accuracy of grammar & -4.7 & .003 & -2.7 & .057 \\
Speed of delivery & -9.2 & $<.000$ & -3.2 & .034 \\
Control of pauses & -8.5 & $<.000$ & -5.3 & .006 \\
Other disfluencies & -6.2 & .001 & -5.0 & .008 \\
Overall fluency & -8.3 & $<.000$ & -24.0 & $<.001$ \\
\hline
\end{tabular}

Summarizing, both main effects (of proficiency and repetition) are significant for all six measures related to accuracy and fluency, giving evidence to the effect of immediate repetition on the improvement of CI performance. It is important to note that, generally, the gain due to repetition is about twice as large for the BA students as for the MA students on fluency-related measures. Significant interaction between proficiency and repetition was found in fluency-related measures. This indicates that BA trainees benefit significantly more from repetition than MA students on fluency-related measures.

\subsection{Linguistic complexity}

The results of the assessment of linguistic complexity are presented in Table 4. 

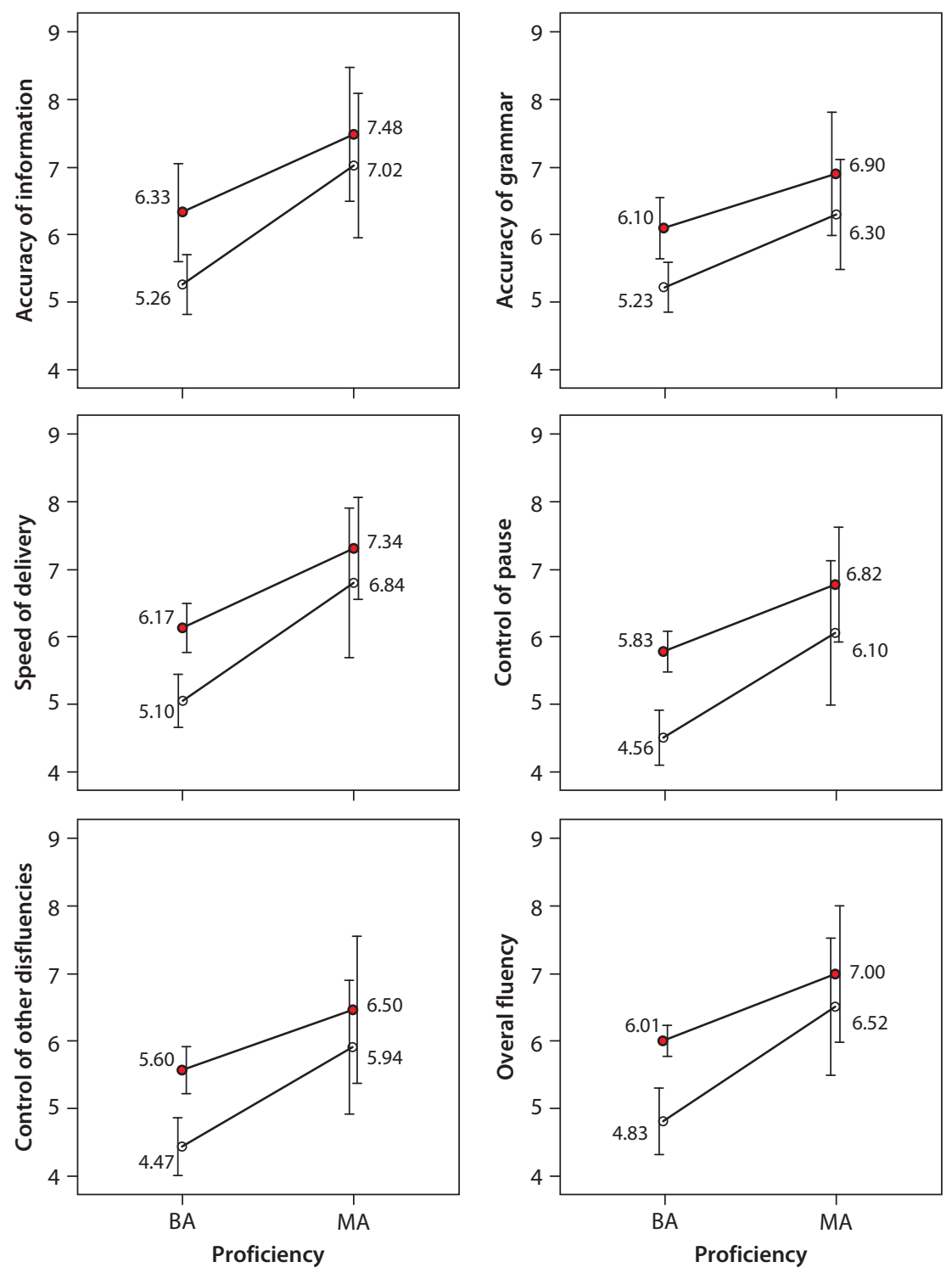

- Delivery $3 \quad$ o Delivery 1

Figure 1. Means and $95 \%$ confidence limits of six measures related to accuracy and fluency as a function of proficiency level (BA level versus MA level) and repetition (Delivery 1 versus Delivery 3) 
Table 4. Complexity (number of clauses per AS unit, further see text).

\begin{tabular}{lcll}
\hline Proficiency & Participants & Complexity & \\
\cline { 3 - 4 } & & Delivery 1 & Delivery 3 \\
\hline BA & 1 & 1.86 & 1.88 \\
BA & 2 & 1.64 & 1.78 \\
BA & 3 & 1.75 & 1.88 \\
BA & 4 & 1.40 & 1.33 \\
BA & 5 & 1.60 & 2.63 \\
BA & 6 & 1.50 & 2.14 \\
BA & 7 & 2.00 & 2.00 \\
Mean BA & & 1.68 & 1.95 \\
MA & 8 & 1.50 & 1.50 \\
MA & 9 & 2.13 & 3.40 \\
MA & 10 & 1.70 & 1.78 \\
MA & 11 & 1.78 & 1.70 \\
MA & 12 & 2.20 & 2.11 \\
Mean MA & & 1.86 & 2.10 \\
Grand mean & & 1.76 & 2.01 \\
\hline
\end{tabular}

The main effect of proficiency on linguistic complexity was absent, $F(1,10)<1$, so was that of repetition, $F(1,10)=3.2\left(p=.105, \mathrm{p} \eta^{2}=.241\right)$. No interaction was found between proficiency and repetition, $F(1,10)<1$.

\section{Discussion and conclusion}

This study investigated the effects of immediate repetition on CI performance at two different training stages (BA level and MA level). The significant main effects of repetition and proficiency show that immediate repetition improves fluency and accuracy of CI performance on a short-term basis. BA trainees made significant improvement on all six measures related to accuracy and fluency in Delivery 3 over Delivery 1 . Almost the same was found for MA trainees except that they improved moderately in accuracy of grammar. Moreover, we observe that BA trainees benefit significantly more from repetition than MA trainees in terms of fluency, which is qualified by the significant interaction between repetition and proficiency. A possible explanation could be that BA trainees, compared with MA trainees, have a weaker link between conceptual content and lexico-grammatical forms as well as weaker form-pronunciation links due to inadequate L2 oral proficiency. Therefore they benefit more from the conceptual, semantic, lexical, syntactic and phonological priming effects from repetition during listening and comprehension, memory 
retrieval, reformulation and articulation stages. The priming effects contribute to fluency and accuracy improvement for both groups, but seem to be more salient on fluency improvement made by BA trainees, which is shown by their significantly larger improvement of fluency compared with MA trainees. MA trainees with higher L2 oral proficiency have stronger links. Therefore, priming yields comparatively less improvement for them in fluency and accuracy of information, and even insignificant improvement in accuracy of grammar, which might be attributed to a fossilization stage before achieving professionalism. Effects of proficiency and repetition on linguistic complexity are absent in this study. Possible reasons could be that the measure used in this study is not sensitive enough, or that the source text does not allow for more complex language, or that improvement of complexity might be a more intensive and longer-term endeavor than that of fluency and accuracy.

The pedagogical implications are that for less proficient interpreting trainees (BA level), immediate repetition is effective on fluency and accuracy improvement by freeing attentional resources through familiarity with content, better memory recall, faster lexico-grammatical retrieval and more automatic articulation. Interpreting trainers need to instruct BA trainees to shift the attentional resources gained from priming to different aspects of interpreting processing. More importantly, BA trainees should avoid striving for impressive fluency at the cost of accuracy of information and grammar. For more proficient interpreting trainees (MA level), more sophisticated training approaches should be designed with a special focus on accuracy of grammar. Further studies with larger sample size are needed to investigate whether the effects of repetition can be transferred to new materials and/or generalize to long-term retention during CI training.

\section{References}

Ahmadian, Mohammad J. 2012. Task repetition in ELT. ELT Journal 66 (3). 380-382.

Ahmadian, Mohammad J. \& Mansoor Tavakoli. 2010. The effects of simultaneous use of careful online planning and task repetition on accuracy, complexity, and fluency in EFL learners' oral production. Language Teaching Research 15. 35-59.

AIIC. 2002. Regulation governing admissions and language classification. AIIC basic texts.

Bühler, Hildegund. 1986. Linguistic (semantic) and extralinguistic (pragmatic) criteria for the evaluation of conference interpretation and interpreters. Multilingua 5. 231-235.

Bygate, Martin. 1996. Effect of task repetition: Appraising the development of second language learners. In J. Willis \& D. Willis (eds.), Challenge and change in language teaching, 136-46. Oxford: Heinemann.

Bygate, Martin. 2001. Effects of task repetition on the structure and control of language. In M. Bygate, P. Skehan \& M. Swain (eds.), Researching pedagogic tasks: Second language learning, teaching and testing, 23-48. Harlow, UK: Pearson Longman. 
Bygate, Martin. 2006. Areas of research that influence L2 speaking instruction. In E. Usó-Juan \& A. Martínez-Flor (eds.), Current Trends in the Development and Teaching of the Four Skills, 159-186. Berlin: Mouton de Gruyter.

Bygate, Martin \& Virginia Samuda. 2005. Integrative planning through the use of task-repetition. In R. Ellis (ed.), Planning and task performance in a second language, 37-74. Amsterdam: Benjamins.

Foster, Pauline \& Peter Skehan. 1996. The influence of planning and task type of second language performance. Studies in Second Language Acquisition 18. 299-323.

Foster, Pauline, Alan Tonkyn \& Gillian Wigglesworth. 2000. Measuring spoken language: A unit for all reasons. Applied Linguistics 21. 354-375.

Freed, Barbara F., Norman Segalowitz \& Dan P. Dewey. 2004. Context of learning and second language fluency in French: Comparing regular classroom, study abroad, and intensive domestic immersion programs. Studies in Second Language Acquisition 26. 275-301.

Gile, Daniel. 1995. Basic concepts and models for interpreter and translator training. Philadelphia: John Benjamins.

Jong, Nel de \& Charles A. Perfetti. 2011. Fluency training in the ESL classroom: An experimental study of fluency development and proceduralization. Language Learning 61. 533-568.

Levelt, Willem J. M. 1989. Speaking: From intention to articulation. Cambridge, MA: MIT Press. Lynch, Tony \& Joan Maclean. 2000. Exploring the benefits of task repetition and recycling in classroom language learning. Language Teaching Research 4. 221-250.

Lynch, Tony \& Joan Maclean. 2001. 'A case of exercising': Effects of immediate task repetition on learners' performance. In M. Bygate, P. Skehan \& M. Swain (eds.) Researching Pedagogic Tasks: Second Language Learning, Teaching and Testing, 141-162. Harlow, UK: Pearson Longman.

Paulston, Christina \& Mary Bruder. 1976. Teaching English as a second language: Techniques and procedures. Cambridge, MA: Winthrop Publishers.

Pavlov, Ivan P. 1957. Experimental psychology and other essays. New York: Philosophical Library.

Segalowitz, Norman \& Barbara F. Freed. 2004. Context, contact, and cognition in oral fluency acquisition. Studies in Second Language Acquisition 26. 173-199.

Skinner, Burrhus F. 1953. S cience and human behavior. New York: Macmillan.

Towell, Richard. 2002. Relative degrees of fluency: A comparative case study of advanced learners of French. International Review of Applied Linguistics in Language Teaching 40. 117-150.

Zhou, Dandan. 2006. A study on the effects of input frequency and output frequency. Modern Foreign Languages 29. 154-163.

Zwischenberger, Cornelia \& Franz Pöchhacker. 2010. Survey on quality and role: Conference interpreters' expectations and self-perceptions. Communicate! http://www.aiic.net/ViewPage. cfm/article2510.htm (21 January, 2013).

\section{Authors' addresses}

Wenting Yu

Shanghai International Studies University

Room 105, No. 88

1880 Longyang Road, Pudong New Area

Shanghai, China 201204

wentingyuyu@gmail.com
Vincent J. van Heuven

Leiden University Centre for Linguistics

P.O. Box 9515

2300 RA Leiden, The Netherlands

v.j.j.p.van.heuven@hum.leidenuniv.nl 